\title{
Successful endovascular embolization for traumatic subcutaneous abdominal wall hematoma via the superficial inferior epigastric artery: a case report
}

\author{
Sung Nam Moon, MD ${ }^{(\mathbb{D}}$, Sang Hyun Seo, MD $\mathbb{B D}^{\circ}$, Hyun Seok Jung, MD (iD \\ Regional Trauma Center, Department of Radiology, Wonkwang University Hospital, Iksan, Korea
}

Received: December 7, 2020

Revised: December 9, 2020

Accepted: December 11, 2020

Correspondence to

Hyun Seok Jung, MD

Department of Radiology, Wonkwang

University Hospital, 895 Muwang-ro,

Iksan 54538, Korea

Tel: +82-63-859-1188

Fax: +82-63-858-3922

E-mail: bighiva@wkuh.org

\begin{abstract}
Abdominal wall hematoma $(\mathrm{AWH})$ after blunt trauma is common, and most cases can be treated conservatively. More invasive treatment is required in patients with traumatic AWH if active bleeding is identified or there is no response to medical treatment. Herein, we report a case of endovascular embolization for traumatic subcutaneous AWH. Almost endovascular treatment for $\mathrm{AWH}$ is done through the deep inferior epigastric artery. However, in this case, the superficial inferior epigastric artery was the bleeding focus and embolization target. After understanding the vascular system of the abdominal wall, an endovascular approach and embolization is a safe and effective treatment option for AWH.
\end{abstract}

Keywords: Abdominal wall; Hematoma; Endovascular procedure; Epigastric artery; Case report

\section{INTRODUCTION}

Abdominal wall injuries are found in approximately $9 \%$ of patients after blunt trauma. Most patients present with lower-grade injuries, ranging from subcutaneous contusion to abdominal wall hematoma (AWH) [1]. Some cases of AWH are accompanied with active bleeding, which may result in serious complications such as anemia, hypovolemic shock, and muscle necrosis from compartment syndrome [2]. Most patients with abdominal wall contusion or hematoma can be managed conservatively with blood transfusion. If conservative management fails, patients might require more invasive treatment [1]. Although no definite treatment guideline for AWH has been established, in recent years the endovascular approach for embolization has been more often used than surgical treatment [3]. We report a case of embo- lization of the superficial inferior epigastric artery (SIEA) for a trauma patient with active subcutaneous bleeding.

\section{CASE REPORT}

A 25-year-old male patient was transported to the emergency department following a motor vehicle accident. On arrival, his vitals were stable (blood pressure, 140/100 $\mathrm{mmHg}$; pulse rate, 85 beats/minute; and respiratory rate, 16 breaths/minute). He was conscious and complained of lower abdominal pain. On abdominal computed tomography, multifocal abdominal wall contusion with focal active bleeding was noted. The active bleeding focus was the subcutaneous layer and anterior aspect of the right rectus abdominis muscle (Fig. 1)

The patient was transferred to the angiography room for endo- 


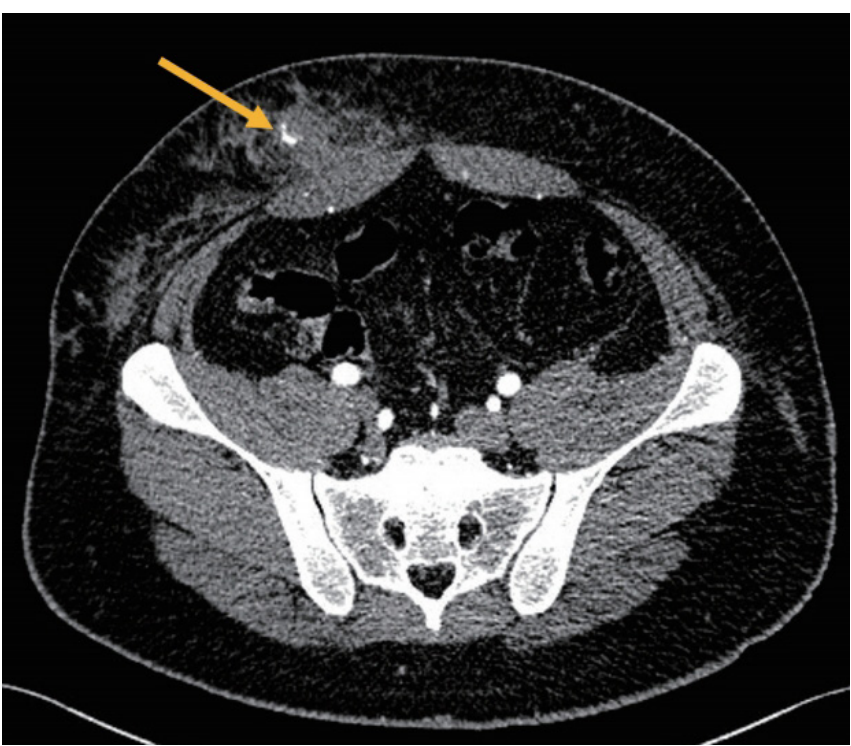

Fig. 1. Contrast-enhanced computed tomography showed subcutaneous abdominal contusion with focal active bleeding (arrow).

vascular treatment. The initial right external iliac arteriogram showed contrast media extravasation (Fig. 2A), but it was not possible to identify the exact culprit vessel. Therefore, the right deep inferior epigastric artery was selected with a microcatheter and an angiogram was done. Contrast extravasation was not noted on the right deep inferior epigastric angiogram, but empirical embolization was done with gelatin sponge particles. A follow-up right external iliac arteriogram showed continuing contrast media extravasation. To find the injury site, an additional selective angiogram was needed. A deep circumflex iliac arteriogram revealed no active bleeding. A secondary selective angiogram was then done for the small branch arising from the medial aspect of the common femoral artery, showing contrast extravasation (Fig. 2B). Embolization was performed for this vessel, the SIEA.

On the 4th hospital day, follow-up computed tomography showed improvement of the abdominal wall contusion without active bleeding (Fig. 3). He was discharged without any complications on that day. The Institutional Review Board waived the requirement for obtaining informed consent due to the retrospective nature of this research.

\section{DISCUSSION}

Blunt trauma can cause various abdominal wall injuries, including contusion, hematoma, traumatic abdominal wall hernia, and Morel-Lavallée lesions, which are closed degloving injuries. It is common for patients to present with AWH [1].

In recent years, the growing number of elderly patients and the
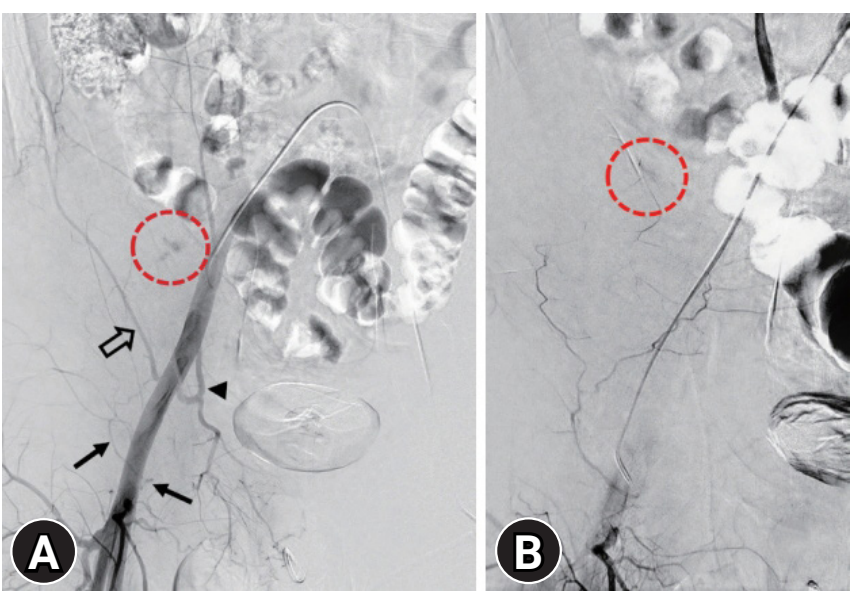

Fig. 2. (A) Right external iliac arteriogram revealed focal contrast extravasation (dotted circle), correlated with the findings of the computed tomography scan, and showed several branches, including the deep inferior epigastric artery (arrowhead), deep circumflex iliac artery (open arrow), and superficial inferior epigastric artery (solid arrows). (B) A selective angiogram of superficial inferior epigastric artery showed focal contrast extravasation (dotted circle).

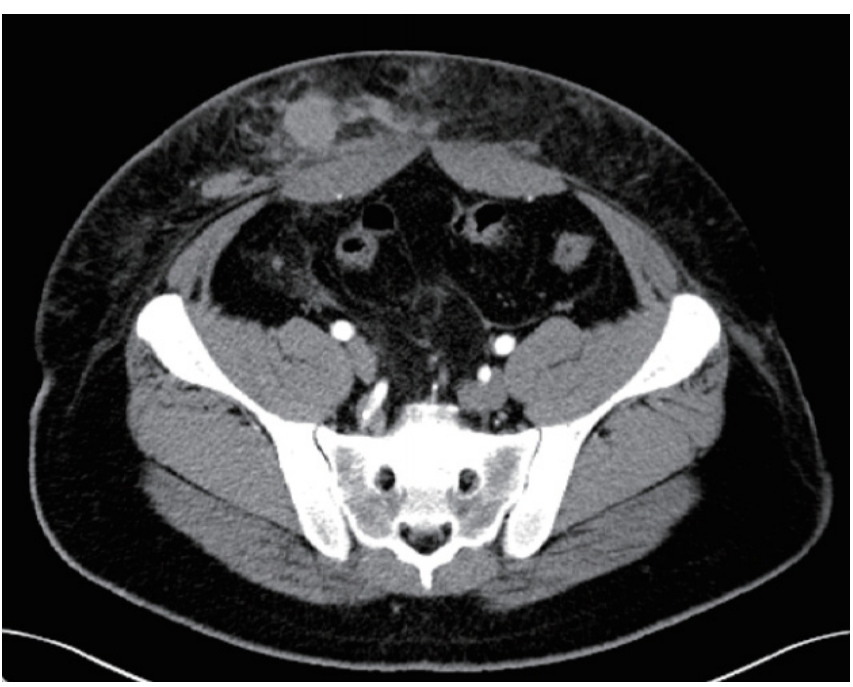

Fig. 3. On the 4th hospital day, follow-up computed tomography showed improvement of the abdominal wall contusion without active bleeding.

increasing exposure of the population to risk factors for hemorrhage such as anticoagulation therapy and chronic hepatopathy have made AWH both more frequent and more dangerous [3]. Medically uncontrolled AWH is an indication for surgical or intravascular intervention to prevent worsening outcomes. Endovascular therapies involving embolization of the injured arteries for hemorrhage control have become a rapid and efficient treatment option, decreasing the morbidity and mortality rates [3,4].

When performing endovascular treatment for $\mathrm{AWH}$, clini- 
cians must be familiar with the vascular system of the anterior abdominal wall. Most cases of AWH involve rectus sheath hematoma, caused by bleeding from the deep inferior epigastric artery. In these cases, the exact bleeding site is the subcutaneous layer of the anterior abdominal wall. The blood supply to the subcutaneous layer of the anterior abdominal wall is variable, but is mainly from the SIEA [5]. In 1975, Taylor et al. [6] described the anatomy of the SIEA. In most people, the SIEA arises from the common femoral artery approximately $1 \mathrm{~cm}$ below the inguinal ligament and ascends in front of the rectus sheath. In approximately one-third of people, the SIEA shares a common origin with the superficial circumflex iliac artery; in another third, the two arteries have separate origins; and in the remaining third, the SIEA is absent [6-8]. After 2010s, as the SIEA flap became more frequently used in plastic and reconstructive surgery, the anatomy of SIEA was more extensively researched using computed tomography images. Several studies reported that the SIEA most commonly has an independent origin from the common femoral artery [8]. In this case, the angiogram showed an independent origin of the SIEA.

In this case, the patient had no significant medical diseases or medication history, and also showed no significant abnormalities on laboratory tests. However, after embolization, the follow-up hemoglobin level decreased by about $2 \mathrm{~g} / \mathrm{dL}$. He did not have any other bleeding focus, excluding AWH. We concluded that endovascular embolization for AWH minimized the drop in hemoglobin and reduced potential complications. Transarterial embolization in patients with traumatic AWH who have active bleeding or do not respond to medical treatment is a safe and effective treatment option, but understanding the configuration of the vascular system prior to the procedure is essential.

\section{NOTES}

\section{Ethical statements}

The Institutional Review Board waived the requirement for obtaining informed consent due to the retrospective nature of this research.

\section{Conflicts of interest}

The authors have no conflicts of interest to declare.

\section{Author contributions}

Conceptualization: all authors; Data curation: all authors; Formal analysis: all authors; Investigation: all authors; Methodology: all authors; Project administration: all authors; Resources: all authors; Software: all authors; Supervision: all authors; Validation: all authors; Visualization: all authors; Writing-original draft: all authors; Writing-review \& editing: all authors.

All authors read and approved the final manuscript.

\section{REFERENCES}

1. Matalon SA, Askari R, Gates JD, Patel K, Sodickson AD, Khurana $B$. Don't forget the abdominal wall: imaging spectrum of abdominal wall injuries after nonpenetrating trauma. Radiographics 2017;37:1218-35.

2. Cherry WB, Mueller PS. Rectus sheath hematoma: review of 126 cases at a single institution. Medicine (Baltimore) 2006; 85:105-10.

3. Albuquerque TV, Monsignore LM, de Castro-Afonso LH, Elias-Junior J, Muglia VF, Abud DG. Transarterial embolization with n-butyl cyanoacrylate for the treatment of abdominal wall hemorrhage. Diagn Interv Radiol 2020;26:216-22.

4. Sobkin PR, Bloom AI, Wilson MW, et al. Massive abdominal wall hemorrhage from injury to the inferior epigastric artery: a retrospective review. J Vasc Interv Radiol 2008;19:327-32.

5. Rozen WM, Chubb D, Grinsell D, Ashton MW. The variability of the Superficial Inferior Epigastric Artery (SIEA) and its angiosome: a clinical anatomical study. Microsurgery 2010; 30:386-91.

6. Taylor GI, Daniel RK. The anatomy of several free flap donor sites. Plast Reconstr Surg 1975;56:243-53.

7. Karunanithy N, Rose V, Lim AK, Mitchell A. CT angiography of inferior epigastric and gluteal perforating arteries before free flap breast reconstruction. Radiographics 2011;31:130719.

8. Reardon CM, O'Ceallaigh S, O'Sullivan ST. An anatomical study of the superficial inferior epigastric vessels in humans. Br J Plast Surg 2004;57:515-9.

9. Fukaya E, Kuwatsuru R, Iimura H, Ihara K, Sakurai H. Imaging of the superficial inferior epigastric vascular anatomy and preoperative planning for the SIEA flap using MDCTA. J Plast Reconstr Aesthet Surg 2011;64:63-8.

Funding

None. 